\title{
Identification of genomic features in the classification of loss- and gain-of-function mutation
}

\author{
Seunghwan Jung ${ }^{1}$, Sejoon Lee², Sangwoo Kim³ ${ }^{3}$ Hojung Nam \\ From ACM Eighth International Workshop on Data and Text Mining in Biomedical Informatics \\ Shanghai, China. 7 November 2014
}

\begin{abstract}
Background: Alterations of a genome can lead to changes in protein functions. Through these genetic mutations, a protein can lose its native function (loss-of-function, LoF), or it can confer a new function (gain-of-function, GoF). However, when a mutation occurs, it is difficult to determine whether it will result in a LoF or a GoF. Therefore, in this paper, we propose a study that analyzes the genomic features of LoF and GoF instances to find features that can be used to classify LoF and GoF mutations.
\end{abstract}

Methods: In order to collect experimentally verified LoF and GoF mutational information, we obtained 816 LoF mutations and $474 \mathrm{GoF}$ mutations from a literature text-mining process. Next, with data-preprocessing steps, 258 LoF and $129 \mathrm{GoF}$ mutations remained for a further analysis. We analyzed the properties of these LoF and GoF mutations. Among the properties, we selected features which show different tendencies between the two groups and implemented classifications using support vector machine, random forest, and linear logistic regression methods to confirm whether or not these features can identify LoF and GoF mutations.

Results: We analyzed the properties of the LoF and GoF mutations and identified six features which have discriminative power between LoF and GoF conditions: the reference allele, the substituted allele, mutation type, mutation impact, subcellular location, and protein domain. When using the six selected features with the random forest, support vector machine, and linear logistic regression classifiers, the result showed accuracy levels of $72.23 \%$, $71.28 \%$, and $70.19 \%$, respectively.

Conclusions: We analyzed LoF and GoF mutations and selected several properties which were different between the two classes. By implementing classifications with the selected features, it is demonstrated that the selected features have good discriminative power.

\section{Background}

A mutation refers to a change of the genomic sequence, which contains all of the genetic information of an organism. Because proteins are generated and regulated based on the genome sequence, alterations of the genome can lead to changes of protein functions [1]. Through these genetic mutations, a protein can loss its native function (loss-of-function), or it can confer a new function (gainof-function) [2-5]. For example, a mutated fumarate

\footnotetext{
* Correspondence: hjnam@gist.ac.kr

'School of Information and Communication Department, Gwangju Institute of Science and Technology, 123 Cheomdangwagi-ro, Buk-gu, Gwangju, Republic of Korea

Full list of author information is available at the end of the article
}

hydratase (FH) loses its native catalytic activity [6], and heterozygous point mutations in isocitrate dehydrogenase (IDH1, IDH2) confer a new metabolic enzymatic activity that produces 2-hydroxyglutarate $[7,8]$. In addition, in the FGFR1 gene, GoF and LoF mutations can lead to different diseases, craniosynostosis and Kallmann syndrome, respectively [9-12]. Therefore, it is important to understand the characteristics of functional mutations and to determine which mutations lead to LoF and GoF results for clinical target.

There are many studies of mutations, including LoF and GoF mutations. MacArthur et al. implemented a systematic survey of LoF variants. They showed many LoF variant properties compared to other mutations, 
such as the allele frequency and the degrees of associations with diseases. They also showed the effects of LoF variants, including phenotypes, diseases, and gene expressions. However, missense mutations were excluded from the LoF mutations which they defined [4]. In our study, many mutations were missense mutations; therefore, we chose to address missense mutations. Reva et al. estimated functional effects of missense mutations using evolutionary conservation information [13]. Lee et al. discussed the bi- directional SIFT (B-SIFT), which is a modified form of SIFT. In addition, the B-SIFT algorithm calculates scores of mutation alleles based on evolutionary conservation information [3]. They used the scores to identify mutations which cause hyperactivation or gain-of-function outcomes, but our work uses not only the functional effects of mutations but also several other properties. However, most previous studies focused on either LoF or GoF mutations or on functional changes in a specific gene.

In this work, we propose a comprehensive analysis of the genomic features in mutations to classify LoF and GoF mutations. Figure 1 shows an overview of our study. First, from the literature, 14,259 gene-sentence relations for GoF and 29,586 relations for LoF were determined. By removing genes without sentences and extracting mutations and their locations from the sentences, we obtained information on $816 \mathrm{LoF}$ and $474 \mathrm{GoF}$ mutations. Next, we applied a data-preprocessing technique. During this process, mutations with an amino acid location were converted into those with a genomic location, and amino acid residues were converted into 3 -mer nucleotide alleles, after which the mutation subtypes were determined. In addition, mutations whose reference allele is not matched with the reference genome as well as mutations published before 2009 were removed. After this processes, $258 \mathrm{LoF}$ mutations and $129 \mathrm{GoF}$ mutations remained. Second, with the remaining mutations, we extracted the features which have discriminative power between LoF and GoF mutations. Lastly, we implemented a classification process using the selected features to confirm whether they can be used to classify the two types. Here, we show six properties of mutations which can contribute to the identification of a LoF or a GoF outcome. These six are the subcellular location, the mutation subtype, the reference and substituted allele, the functional impact, and the protein domain.

\section{Method}

\section{Text mining}

Mutation information with the experimentally characterized LoF and GoF outcomes was collected from the literature. In this case, we extracted information about LoF and GoF outcomes from PubMed. First, we searched all
PubMed abstracts which contain the acronyms "GOF" and "LOF" and the words "gain of function," "gain-offunction," "loss of function" and "loss-of-function" as keywords. Then, we found all related genes for each abstract and sentence containing the relevant genes using Gene2Pubmed [14]. Next, we tagged the mutations and their locations using tmVar, which is a previously published software, to extract mutation information from the literature : substitutions, insertions, deletions, and SNP and frameshift mutations of DNA and protein sequences, so we also used CRF features as mentioned in the reference [15].

\section{Data preprocessing}

Once the LoF and GoF mutation information was collected from the literature, the mutation data set was preprocessed for further analysis. First, we selected LoF and GoF mutations that were published after the year 2010 in order to filter out mutations which had been identified against older versions of the reference genome. Second, mutations that are represented with an amino acid information were converted into those with a genomic location using the exon and intro information in Consensus CDS (CCDS, using GRCh37.p13) [16-18] so that we could observe the differences between LoF and GoF mutations at the nucleotide sequence level. Also, amino acid residues were converted into 3-mer nucleotide alleles by incorporating the CCDS nucleotide sequence and the amino acid codon table. In addition, substituted mutations were subgrouped into missense, nonsense and silent mutations according to the amino acid residue. During this step, silent mutations were removed. After these preprocessing steps, 258 LoF mutations and 129 GoF mutations remained.

\section{Mutation impact}

In order to evaluate the significance of missense mutation effects on protein functions, the functional impact scores (FIS) of the LoF and GoF mutations were evaluated using the FIS method [13], which calculates the significance scores of point mutations based on evolutionary conservation of the mutation sites. We used the following information as the input of the FIS method: hg version, chromosome, mutation location, reference allele, substituted allele.

\section{Protein domain}

To demonstrate the relationships between the protein domain functions and the LoF and GoF mutations, we used the Pfam database [19], which makes available large-scale protein domain information about proteins. We found protein domains corresponding to LoF and GoF mutation locations and formulated a distribution of the protein domains of the two classes with all 55931 


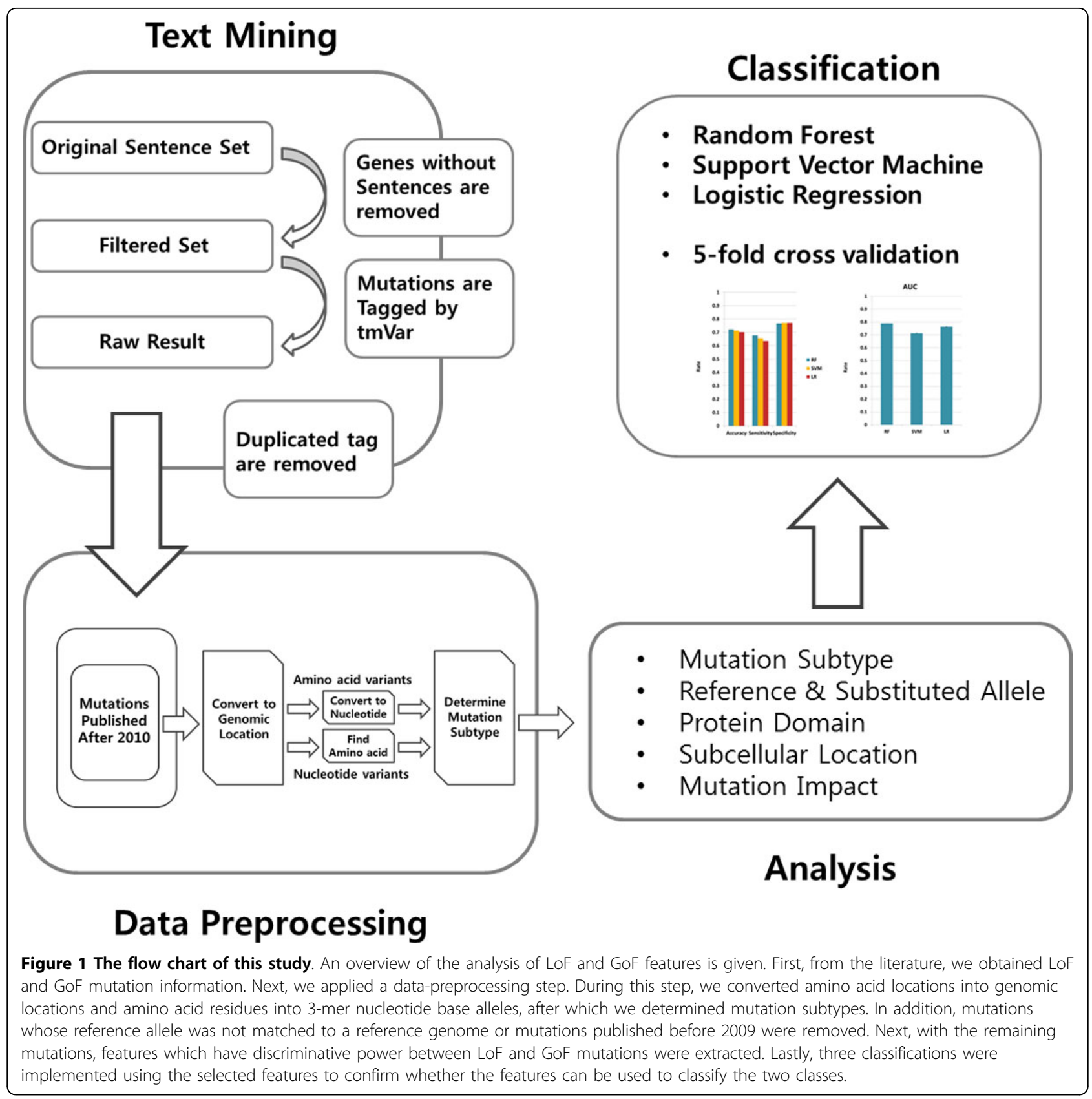

human genes as the background. With this information, we performed a hypergeometric test to find the protein domains which had significantly different distributions.

\section{Machine learning}

In order to classify LoF and GoF mutations based on our defined features, we utilized the support vector machine (SVM) with normalized poly kernel, random forest (RF) with 100 trees, logistic regression methods using the WEKA package $[20,21]$. Both SVM and logistic regression use linear decision boundaries, but unlike the logistic regression, not all instances affect building classifier function in the SVM. Only instances near the boundary are considered, and such difference between two classifiers can lead to different result. Six features were selected to build a classifier: the reference allele, the substituted allele, the mutation type, the mutation impact, the subcellular location and the protein domain. Among these features, the mutation impacts are continuous attributes, and the other features are nominal attributes. Then, WEKA package automatically transformed nominal attributes into binary ones if classifier cannot handle the nominal attributes. To avoid bias due to the different data sizes in the two classes, we randomly chose 100 
instances of mutation data from each of the LoF and GoF data sets, after which the random selections were iterated 50 times, so 50 equal size data sets were made. For each data set, we performed five-fold cross validation and repeated the five-fold cross validation process 100 times. For each five-fold cross validation, 160 instances were used to train the classifiers, and 40 instances were used to test. As a result, we obtained 5,000 five-fold cross validation results. Table 1 shows an example of input instances.

\section{Results}

\section{Tagged mutations from the literature}

We obtained 14,259 gene-sentence relationships for GoF and 29,586 relationships for LoF. From these relationships, genes which did not have sentences were removed. We obtained 2,142 sentences for GoF and 4,600 sentences for LoF as a result. Next, tmVar [15] found 474 mutations for GoF and 816 mutations for LoF. Consequently, we obtained 474 mutations from 2,142 sentences for GoF and 816 mutations from 4,600 sentences for GoF.

\section{Overlapping genes}

First, from the literature, we obtained mutation information for $816 \mathrm{LoF}$ and $474 \mathrm{GoF}$ mutations. Next, during the data-preprocessing step, mutations whose reference alleles were not matched with a reference genome or mutations published before 2009 were removed. As a result, there remained 258 LoF mutations and 129 GoF mutations. We extracted gene names from the 258 LoF mutations and 129 GoF mutations. Finally, 109 LoF genes and 59 GoF genes were selected for further analysis. Figure 2 demonstrates that there were 15 common genes. However, since the gene names are distributed broadly, there is no pattern that can be used to classify LoF and GoF mutations.

\section{Subcellular location}

The subcellular location information of the proteins was collected from the UniProt database [22]. We then analyzed the enriched subcellular locations of the LoF and GoF mutant genes using a hypergeometric test against the information of the total of 22119 subcellular locations of human proteins. Figure 3A shows the calculated distributions of the subcellular locations of the LoF and

Table 1. Input format example

\begin{tabular}{lllllll}
\hline REF & SUB & TYPE & SCORE & SL & PD & Class \\
\hline A & G & Missense & 1.78 & Cell membrane & Cadherin & GoF \\
\hline $\mathrm{T}$ & $\mathrm{G}$ & Missense & 3.62 & Isoform2 & SelR & LoF \\
\hline $\mathrm{T}$ & $\mathrm{A}$ & Missense & -1.505 & Apical cell membrane & ASC & LoF \\
\hline
\end{tabular}

Six features were used: the reference allele (REF), the substituted allele (SUB), the mutation type (TYPE), the mutation impact (SCORE), the subcellular location (SL) and the protein domain (PD).

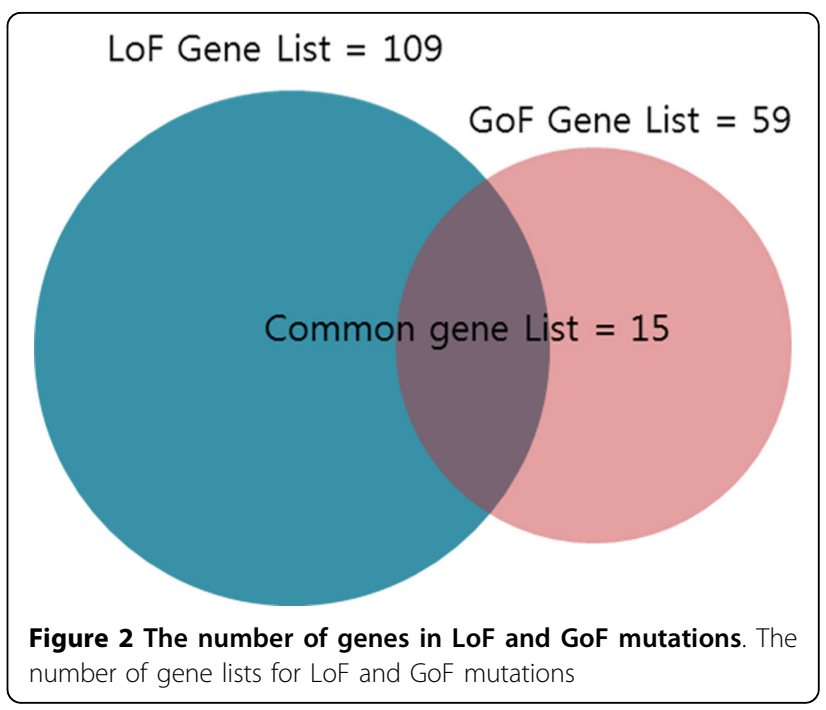

GoF genes, including in each case the nucleus, cell membrane, cytoplasm, membrane, and secreted. The subcellular location which contains the highest number of LoF mutated genes is the nucleus (19.61\%), while in GoF it is the cell membrane (23.44\%). When we implemented the hypergeometric test to compare the distributions between the LoF and the human results, and between the GoF and the human results, we found that the distributions of the subcellular locations of LoF mutations and the background subcellular locations were significantly different in the cell membrane and cytoplasm (P-value $=0.0159,0.0180)$. In addition, the distributions of the subcellular locations of the GoF mutations and the background subcellular locations were significantly different in the nucleus, cell membrane, and membrane $(\mathrm{P}$-value $=0.0356,0.0001,0.0254$, respectively).

\section{Mutation subtypes}

Next, we extracted mutation subtypes from the LoF and GoF mutations and compared their distributions. In this work, we used six types of mutations: missense, nonsense, deletion, indel, duplication and frame shift. Figure 3B shows the distribution of the mutation subtypes of LoF and GoF. MacArthur et al. studied LoF variants, but did not focus on the missense mutations [4]. However, our study shows that the most frequently found type of mutation is the missense mutation in both cases for LoF and GoF mutations. This ratio indicates that missense mutations are also an important proportion of the mutations which affect protein functions. The second most frequently found mutation is the nonsense mutation in LoF; for GoF, it was the deletion mutation. These results indicate that nonsense mutations usually lead to a protein which causes a loss of function and not a gain of a new function. 


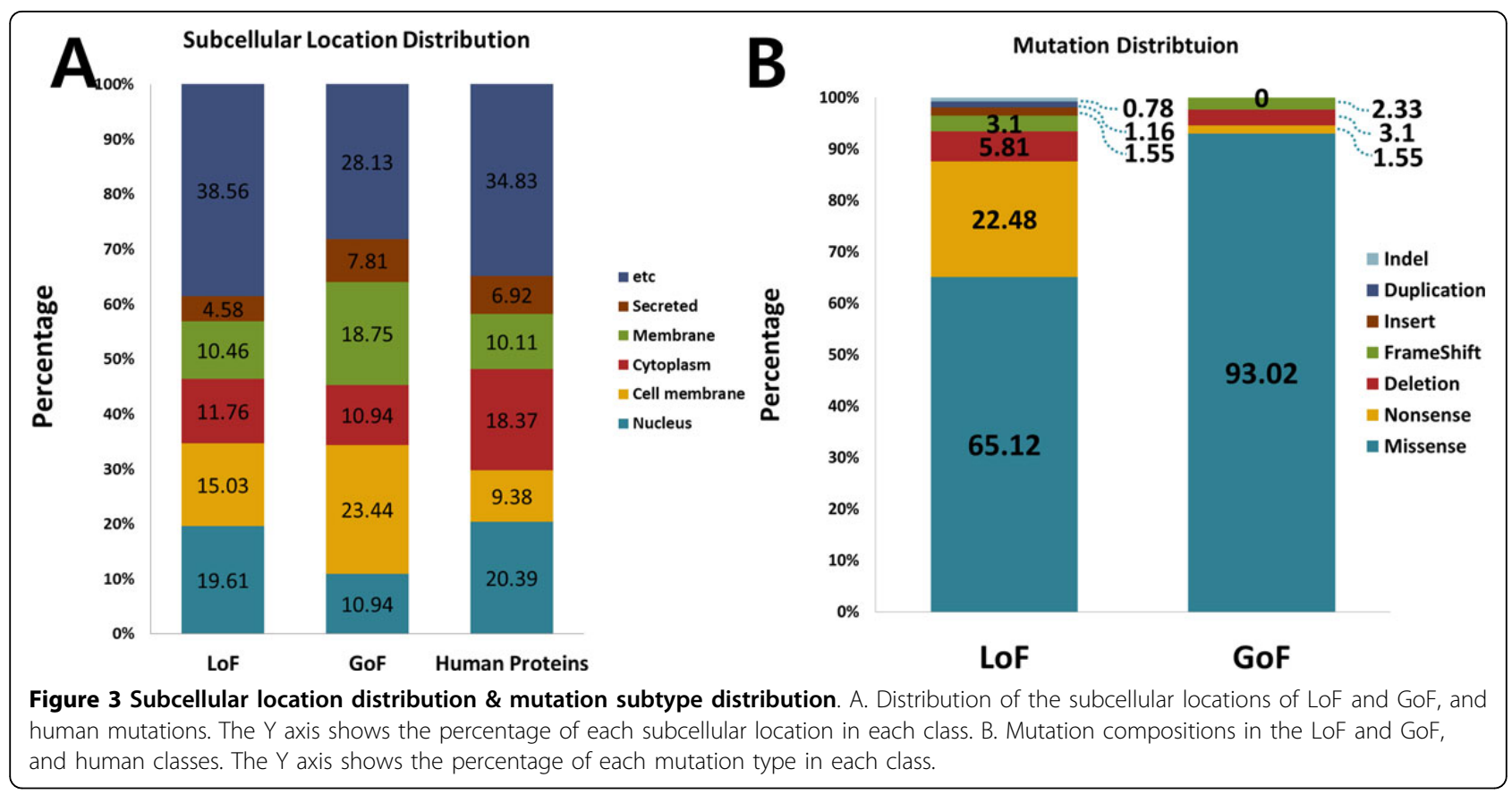

\section{Reference and substituted allele ratio}

We extracted the nucleotide reference alleles and substituted alleles and classified allele pairs into two groups based on their nitrogenous bases. If a reference allele and a substituted allele were of the same nitrogenous base (purine and purine, pyrimidine and pyrimidine), the mutation was classified as a transition (Ti). Otherwise, if the two nitrogenous bases were different, it was classified as a transversion (Tv). We then analyzed the differences in the proportions of the each allele pairs between the LoF and GoF mutations. Figure 4 shows the ratio of reference and the substituted allele pairs, and Table 2 describes P-values pertaining to the result of comparing the LoF and GoF mutations using the propositional binomial. There were no significant differences found in the TiTv ratio between LoF and GoF, but for the LoF case, the transition (Ti) percentage is higher than the transversion ( $\mathrm{Tv}$ ) percentage. In addition, the AG, CT, AT, and GT results show significant differences (P-values: 0.0347, 0.0376, 0.0426, and 0.0399, respectively).

\section{Protein domain}

We collected the protein domain function information of each mutation. Figure 5 shows the distribution of the protein domain functions of the LoF and GoF mutations. The protein domain functions are distributed broadly, but some of them show several exclusive cases between the two classes. This result indicates that LoF and GoF mutations tend to affect different protein functions. Next, we analyzed the protein domain functions of the LoF and GoF mutations using a hypergeometric test against 55931 human protein domain functions.

\section{Mutation impact}

The FIS method was used to estimate the significance of the missense mutation effects on the protein functions, classifying mutations into four grades based on the estimated scores: high, medium, low, and neutral [13]. Mutations classified as a higher grade mutation have a more of an effect on protein functions than those classified as a lower grade mutation. Figure 6 shows the distributions of the functional impact grades of the LoF and GoF mutations. The percentages differ in high-impact mutations as compared to low-impact mutations. The LoF results shows a higher percentage of high-impact mutations than the GoF results (LoF: $24.49 \%$, GoF: $14.81 \%$ ) as well as a lower percentage in low-impact mutations (LoF: $20.41 \%$, GoF: $29.63 \%)$. This result indicates that LoF mutations affect the protein function more than GoF mutations.

\section{Classification of LoF versus GoF with selected features}

To confirm whether or not the properties can be used as criteria for distinguishing LoF and GoF mutations, we implemented a classification technique using the support vector machine, random forest, and linear logistic regression methods with 50 data sets which contain equal numbers of LoF and GoF mutations to avoid bias. As a result of five-fold cross validation repeated 100 times, we obtained 25,000 results for each classifier and calculated the averages of the total accuracy, true positive rates (rates of LoF correctly classified as LoF), and 


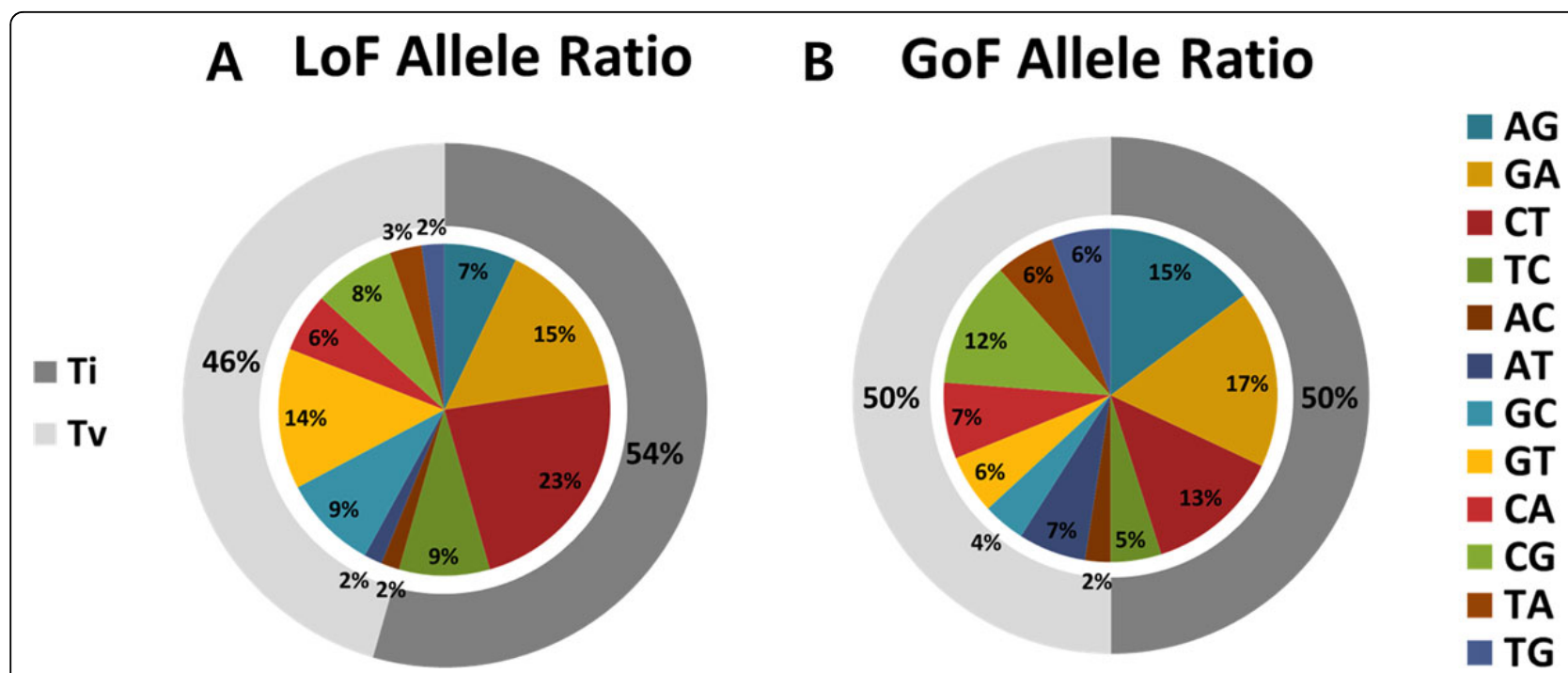

Figure 4 Allele. A. Reference to substituted allele rates and transition and transversion (Ti, TV) rates in LoF. B. Reference to substituted allele rates and transition and transversion (Ti, TV) rates in GoF.

true negative rates (rates of GoF correctly classified as GoF). Figure 7A shows the accuracy rates, the true positive rates (sensitivity), and the true negative rates (specificity); while Figure $7 \mathrm{~B}$ shows the AUC rates. The average percent correctly classified was $71.28 \%$ for the support vector machine method, $72.23 \%$ for the random forest method, and $70.19 \%$ for the linear logistic regression method, while the AUC values for each classification were $0.7128,0.7880$, and 0.7646 . From these results, we can confirm the discriminative power of the six features.

Table 2. Allele P-value

\begin{tabular}{ll}
\hline Allele Pair & P-value \\
\hline AG & 0.0347 \\
\hline GA & 0.7908 \\
\hline CT & 0.0376 \\
\hline Transition & 0.2639 \\
\hline AC & 0.4987 \\
\hline AT & 0.9707 \\
\hline GC & 0.0426 \\
\hline GT & 0.1224 \\
\hline CA & 0.036 \\
\hline CG & 0.7162 \\
\hline TA & 0.261 \\
\hline TG & 0.3627 \\
\hline Transversion & 0.158 \\
\hline P-valus from the compaison & 0.4987
\end{tabular}

P-values from the comparison of the proportions of 12 references, i.e., the substituted allele pairs and the transition (Ti) and transversion (Tv) ratios between the LoF and GoF mutations.

\section{Conclusion and Discussion}

By mining the literature, 14,259 gene-sentence relationships for GoF and 29,586 relationships for LoF were collected. From these, genes without sentences were removed. Thus, 2,142 sentences for GoF and 4,600 sentences for LoF remained. We then tagged the mutations and their locations from the sentences. Consequently we obtained 474 mutations from 2,142 sentences for GoF and 816 mutations from 4,600 sentences for GoF. In addition, during the data-preprocessing step, mutations whose reference allele was not matched with a reference genome or mutations published before 2009 were removed. Hence, we analyzed $258 \mathrm{LoF}$ mutations and $129 \mathrm{GoF}$ mutations. As a result, we found six features which can distinguish LoF and GoF mutations: the subcellular location, the mutation subtype, the reference and substituted allele, the functional impact, and the protein domain. We used these features for classification to confirm whether or not they can identify LoF and GoF mutations. Finally, we obtained $72.23 \%$ accuracy for the random forest, $71.28 \%$ accuracy for the support vector machine, and $70.19 \%$ accuracy for the linear logistic regression methods, with AUC values of $0.7880,0.7128$, and 0.7646 , respectively. As a result, we can conclude that the selected features can contribute to the identification of LoF and GoF mutations.

\section{Limitations and Future Work}

Since the LoF and GoF mutation data were derived from the literature, the number of mutation data was limited and was not enough to understand overall tendency of the LoF and GoF mutations. In addition, although we selected mutations that were published after the year 2010, there were mutations not matched with the reference genome. 


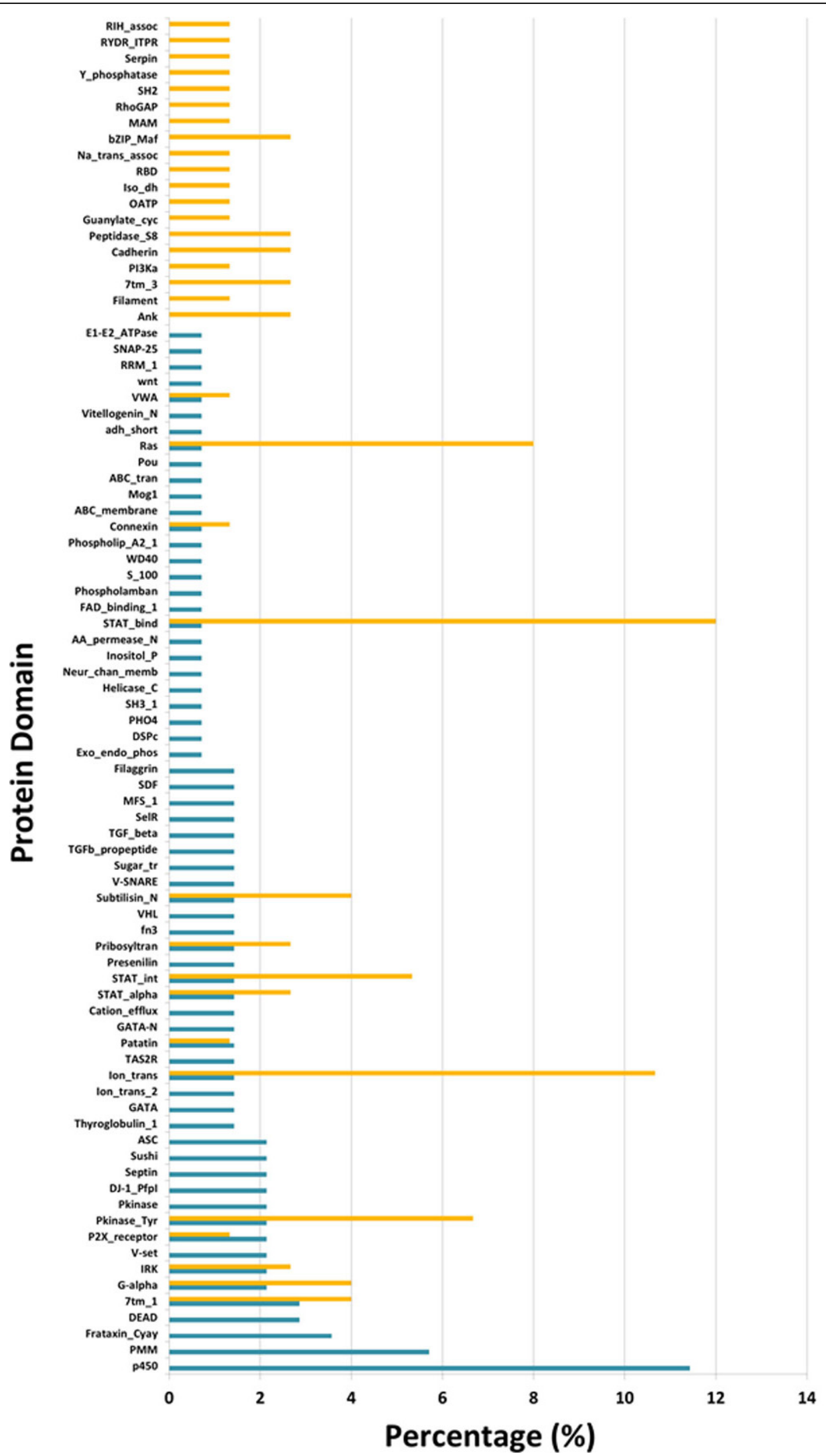

GoF

Figure 5 Protein Domain. LoF and GoF rates in protein domains 


\section{Functional Impact Distribution}

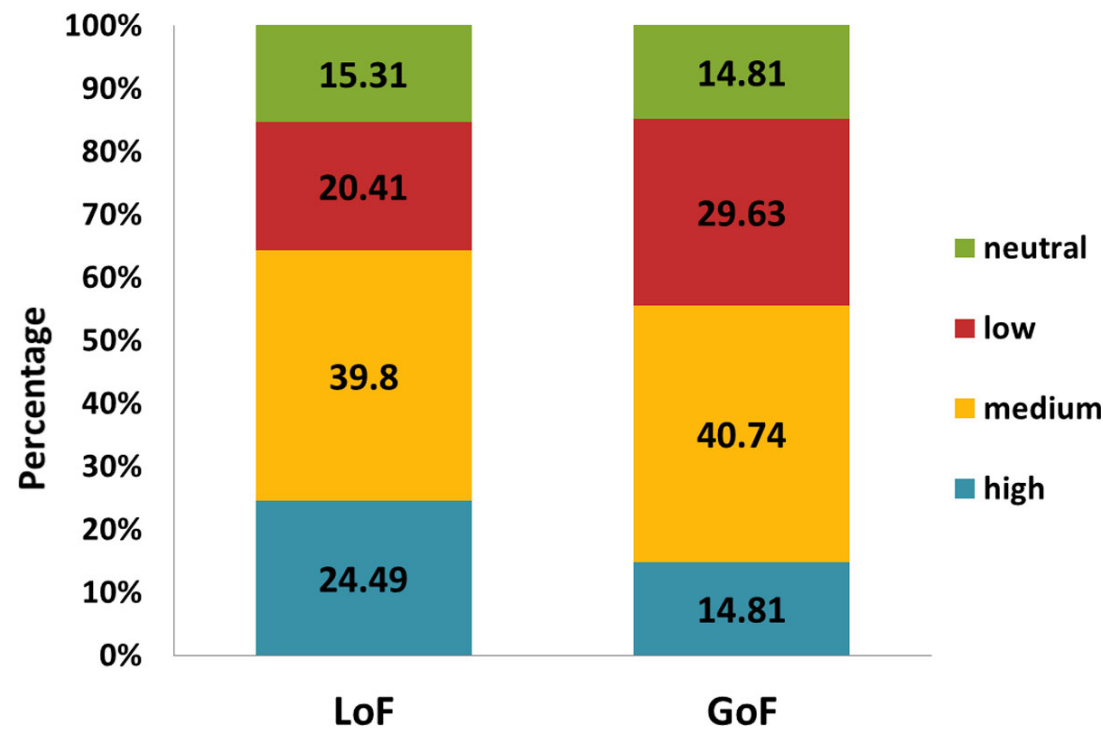

Figure 6 Mutation Impact. Distribution of the functional impact of missense mutations. The functional impact is the estimation how much the mutation affects the protein function. The $Y$ axis shows the percentage of each functional impact in each class.

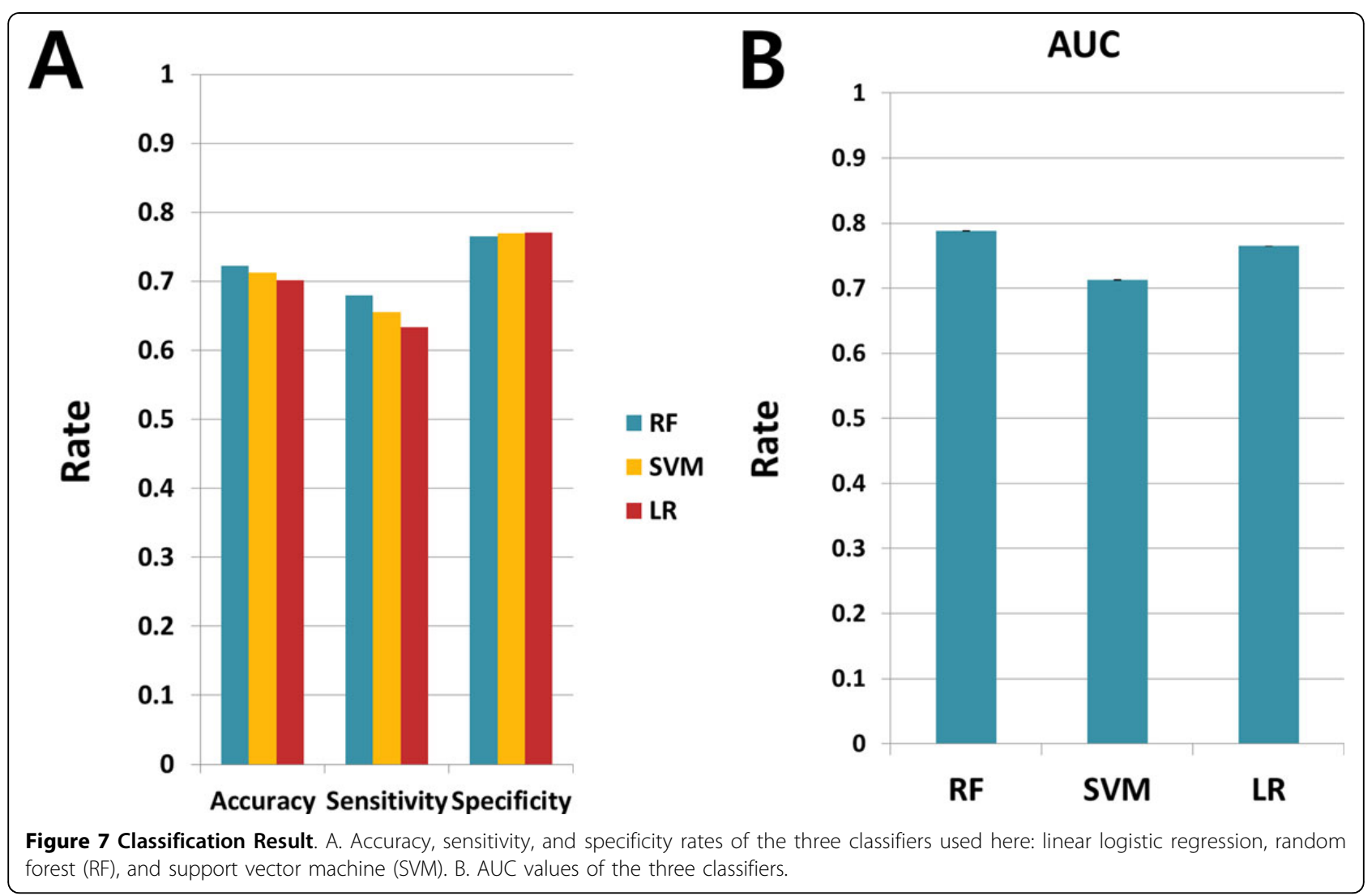


In this work, we studied the LoF and GoF mutation properties, and we expect that this study can contribute to better understanding of the mutation effects on the biological systems. Through the analysis of associations between mutations and protein functions and the analysis of how the affected proteins influence the biological pathways, we can clarify biological mechanism from mutations to systems.

\section{Competing interests}

The authors declare that they have no competing interests.

\section{Authors' contributions}

SJ conducted the analysis and drafted the manuscript. HN and SK designed and coordinated the study. SL performed the literature data mining.

\section{Acknowledgements}

This work was supported by GIST College's 2014 GUP Research Fund and by the "Systems Biology Infrastructure Establishment Grant" provided by the Gwangju Institute of Science \& Technology in 2014.

\section{Declaration}

Publication costs for the article were sourced from GIST College's 2014 GUP Research Fund and by the "Systems Biology Infrastructure Establishment Grant" provided by the Gwangju Institute of Science \& Technology in 2014. This article has been published as part of BMC Medical Informatics and Decision Making Volume 15 Supplement 1, 2015: Proceedings of the ACM Eighth International Workshop on Data and Text Mining in Biomedical Informatics. The full contents of the supplement are available online at http://www.biomedcentral.com/bmcmedinformdecismak/supplements/15/S1.

\section{Authors' details}

'School of Information and Communication Department, Gwangju Institute of Science and Technology, 123 Cheomdangwagi-ro, Buk-gu, Gwangju, Republic of Korea. ${ }^{2}$ Bio-Synergy Research Center, Daejeon, Republic of Korea. ${ }^{3}$ Severance Biomedical Science Institute, Yonsei University College of Medicine, Seoul 120-752, Korea.

\section{Published: 20 May 2015}

\section{References}

1. Kandoth C, McLellan MD, Vandin F, Ye K, Niu B, Lu C, Xie M, Zhang Q, McMichael JF, Wyczalkowski MA, et al: Mutational landscape and significance across 12 major cancer types. Nature 2013, 502(7471):333-339

2. Benjannet S, Hamelin J, Chretien M, Seidah NG: Loss- and gain-of-function PCSK9 variants: cleavage specificity, dominant negative effects, and low density lipoprotein receptor (LDLR) degradation. The Journal of biological chemistry 2012, 287(40):33745-33755.

3. Lee W, Zhang Y, Mukhyala K, Lazarus RA, Zhang Z: Bi-directional SIFT predicts a subset of activating mutations. PloS one 2009, 4(12):e8311.

4. MacArthur DG, Balasubramanian S, Frankish A, Huang N, Morris J, Walter K, Jostins L, Habegger L, Pickrell JK, Montgomery SB, et al: A systematic survey of loss-of-function variants in human protein-coding genes. Science 2012, 335(6070):823-828.

5. Strano S, Dell'Orso S, Mongiovi AM, Monti O, Lapi E, Di Agostino S, Fontemaggi G, Blandino G: Mutant p53 proteins: between loss and gain of function. Head \& neck 2007, 29(5):488-496.

6. Xiao M, Yang H, Xu W, Ma S, Lin H, Zhu H, Liu L, Liu Y, Yang C, Xu Y, et al: Inhibition of alpha-KG-dependent histone and DNA demethylases by fumarate and succinate that are accumulated in mutations of $\mathrm{FH}$ and SDH tumor suppressors. Genes \& development 2012, 26(12):1326-1338.

7. Lu C, Ward PS, Kapoor GS, Rohle D, Turcan S, Abdel-Wahab O, Edwards CR, Khanin R, Figueroa ME, Melnick A, et al: IDH mutation impairs histone demethylation and results in a block to cell differentiation. Nature 2012, 483(7390):474-478
8. Xu W, Yang H, Liu Y, Yang Y, Wang P, Kim SH, Ito S, Yang C, Wang P, Xiao MT, et al: Oncometabolite 2-hydroxyglutarate is a competitive inhibitor of alpha-ketoglutarate- dependent dioxygenases. Cancer cell 2011, 19(1):17-30.

9. Dode C, Levilliers J, Dupont JM, De Paepe A, Le Du N, Soussi-Yanicostas N, Coimbra RS, Delmaghani S, Compain-Nouaille S, Baverel F, et al: Loss-offunction mutations in FGFR1 cause autosomal dominant Kallmann syndrome. Nature genetics 2003, 33(4):463-465.

10. Hu Y, Bouloux PM: Novel insights in FGFR1 regulation: lessons from Kallmann syndrome. Trends in endocrinology and metabolism: TEM 2010 21(6):385-393.

11. Ibrahimi OA, Zhang F, Eliseenkova AV, Linhardt RJ, Mohammadi M: Proline to arginine mutations in FGF receptors 1 and 3 result in Pfeiffer and Muenke craniosynostosis syndromes through enhancement of FGF binding affinity. Human molecular genetics 2004, 13(1):69-78.

12. Zhou YX, Xu X, Chen L, Li C, Brodie SG, Deng CX: A Pro250Arg substitution in mouse Fgfr1 causes increased expression of Cbfa1 and premature fusion of calvarial sutures. Human molecular genetics 2000, 9(13):2001-2008

13. Reva B, Antipin $Y$, Sander C: Predicting the functional impact of protein mutations: application to cancer genomics. Nucleic acids research 2011, 39(17):e118.

14. Maglott D, Ostell J, Pruitt KD, Tatusova T: Entrez Gene: gene-centered information at NCBI. Nucleic acids research 2007, 35(Database):D26-31.

15. Wei $\mathrm{CH}$, Harris $\mathrm{BR}$, Kao HY, Lu Z: tmVar: a text mining approach for extracting sequence variants in biomedical literature. Bioinformatics 2013, 29(11):1433-1439.

16. Farrell CM, O'Leary NA, Harte RA, Loveland JE, Wilming LG, Wallin C, Diekhans M, Barrell D, Searle SM, Aken B, et al: Current status and new features of the Consensus Coding Sequence database. Nucleic acids research 2014, 42(Database):D865-872.

17. Harte RA, Farrell CM, Loveland JE, Suner MM, Wilming L, Aken B, Barrell D, Frankish A, Wallin C, Searle $S$, et al: Tracking and coordinating an international curation effort for the CCDS Project. Database : the journal of biological databases and curation 2012, 2012:bas008.

18. Pruitt KD, Harrow J, Harte RA, Wallin C, Diekhans M, Maglott DR, Searle S, Farrell CM, Loveland JE, Ruef BJ, et al: The consensus coding sequence (CCDS) project: Identifying a common protein-coding gene set for the human and mouse genomes. Genome research 2009, 19(7):1316-1323.

19. Finn RD, Bateman A, Clements J, Coggill P, Eberhardt RY, Eddy SR, Heger A, Hetherington K, Holm L, Mistry J, et al: Pfam: the protein families database. Nucleic acids research 2014, 42(Database):D222-230.

20. Bakheet TM, Doig AJ: Properties and identification of human protein drug targets. Bioinformatics 2009, 25(4):451-457.

21. Hall $M$, Frank E, Holmes $G$, Pfahringer $B$, Reutemann $P$, Witten IH: The WEKA data mining software: an update. SIGKDD Explor News/ 2009, 11(1):10-18.

22. UniProt C: Activities at the Universal Protein Resource (UniProt). Nucleic acids research 2014, 42(Database):D191-198, [1-22].

doi:10.1186/1472-6947-15-S1-S6

Cite this article as: Jung et al.: Identification of genomic features in the classification of loss- and gain-of-function mutation. BMC Medical Informatics and Decision Making 2015 15(Suppl 1):S6.

\section{Submit your next manuscript to BioMed Central and take full advantage of:}

- Convenient online submission

- Thorough peer review

- No space constraints or color figure charges

- Immediate publication on acceptance

- Inclusion in PubMed, CAS, Scopus and Google Scholar

- Research which is freely available for redistribution 